Chemie eindringen zu sehen, aber uns fehlt bisher eine historisch entwickelte struktur-theoretische Behandlung der allgemeinen organischen Chemie, die sich, wo angängig, auf physikalisch-chemischen Boden stellt. Der Verfasser fullit die Lücke in einer für beide Teile erfreulichen Weise aus, für den Organiker, welcher allgemeine Gesichtspunkte kennen lernen, und den Physiko-Chemiker, der sich in die Anschaungen der Organiker einarbeiten will. - Wenn, der Natur des Gegenstandes entsprechend, das Bestreben, die physikalisch-chemischen Anschauungen in den Vordergrund zu stellen, nur in wenigen Kapiteln in breiterer Darstellung hervortritt, so wirkt es an diesen Stellen doch um so überzeugender, da wir dann deutlich sehen, wie festgeschlossen die Gesetze der physikalischen Chemie den mehr qualitativen und formalen Spekulationen der reinen Organiker gegenibber stehen.

Bei einer Neubearbeitung wird es sich doch empfehlen, noch einige Kapitel der organischen Chemie mehr zu berïcksichtigen, welche diesmal - der Verfasser war im Raume beschränkt - kein Unterkommen haben finden können. Ich denke dabei in erster Linie an die Arbeiten ther katalytische Beschleunigung und Hemmung bei Oxydations- und Reduktionsprozessen und an die Arbeiten über die elektrochemische Reduktion der Nitroderivate. Zählen doch gerade diese letzteren zu den ganz wenigen, bei denen es gelungen ist, einfach erscheinende Vorgänge in eine lange komplizierte Folge von Zwischenreaktionen zu zerlegen, und bei denen die Aufklärung der einzelnen Phasen ausschließliches Verdienst der physikalischen Chemie ist. Askenasy.

Prinziplen der Chemle. Eine Einleitung in alle chemischen Lehrbücher. Von WIlh. Ostwald. XV und 540.S. mit 65 Figuren. Verlag der Akademischen Verlagsgesellschaft m. b. H., Leipzig. 1907. Preis brosch. $8 \mathrm{Mk}$, geb. 8,80 Mk.

Wer nach dem Untertitel dieses Werkes glauben wollte, $\mathrm{dab}$ es sich hier um eine leichte einfthrende Lektlltre handelt, würde sich gründlich täuschen. Es handelt sich vielmehr um eine erkenntnistheoretische Herausarbeitung 80 ziemlich aller Begriffe und Definitionen, mit denen der Chemiker mehr oder weniger sicher zu operieren lernt, meist ohne daß er sich über ihre Genesis und ihre Grundlagen klar wird. Es ist im Rahmen einer Besprechung nicht möglich, auf Einzelheiten einzugehen, sondern es muß der Hinweis genügen, daB wohl jedem Leser dieses Buches mit Verwunderung oder Beschämung zum Bewußtsein kommen wird, wie viel er in dieser Richtung als selbstverständlich betrachtet, d. h. undurchdacht gelassen hat.

Der Inhalt zerfällt in die Kapitel: I. Körper, Stoffe und Eigenschaften, 2. die Formarten, 3. Gemenge, Lösungen und reine Stoffe, 4. Unwandlung der Formarten und Gleichgewichte. 5. Lösungen, 6. Elemente und Verbindungen, 7. das Gesetz der Verbindungsgewichte, 8. die kolligativen Eigenschaften, 9. Reaktionsgeschwindigkeit und Gleichgewicht, Io. Isomerie, II. die Ionen.
Neben der überall hervortretenden besonderen Sorgfalt in der Präzisierung bekannter Begriffe sind in vielen Stellen auch ganz neuartige Darstellungen eingeführt worden. Dahin gehört vor allem die graphisch versinnbildlichte Systematik der binären Gleichgewichte in 5. und 6. Kapitel.

Man kann sich leicht vorstellen, daß eine Darstellung der chemischen Gesetze ohne Bezugnahme auf die speziellen Stoffe an die Vorstellungskraft des Lesers hohe Anforderungen stellt, und es gehört die ganze stilistische Kunst eines Ostwald dazı, um diesem "Knochengerüst des chemischen Körpers" ein erfreu. liches Exterieur $z \mathfrak{u}$ verleihen. Immerhin sieht sich der Leser gleich einem Paläontologen dauernd vor der mehr oder weniger mühsamen, wenn auch reizvollen Aufgabe, dieses Skelett mittels lebendiger chemischer Tatsachen zu dem Lebewesen Chemie zu rekonstruieren.

Die kurze Reihe der auf $S$. XV vermerkten, sachlich meist gänzlich unerheblichen Korrigenda. könnte leicht auf den fünffachen Umfang gebracht werden, wenn es sich überhaupt lohnte, in wissenschaftlichen Werken Fehler, wie „klen" statt ,"klein ", zu erwähnen.

Alles in allem hat man es mit einem Buche zu tun, das, wie alle Ostwaldschen, ausgeprägte Eigenart und dementsprechenden Reiz für jeden Leser besitzt. Namentlich allen denen, die als Lehrer unserer Wissenschaft die Aufgabe haben, ihre Schüler in die Anfangsgrïnde einzuführen, wird dieses Werk wichtige und willkonımene Hilfe dafür bieten, wie auf moderner Basis eine solche Einführung folgerichtig und unter gebuhrender Trennung von Hypothese und Erfahrung gestaltet werden kann.

R. A begg.

Collection of papers contributed on the occasion of the celebration of Prof. J.Sakurals jubilee, reprinted from the Journal of the College of Science. Vol. XXV. Tokyo, August Igo8. Mit einem Bild Sakurais.

Diese Sammlung zu Ehren des verdienten Vorkämpfers der physikalischen Chemie in Japan ist zu seinem 25jährigen Professorenjubiläum und 50 . Geburtstag von seinen Schtilern herausgegeben worden. Unter den I8 Artikeln seien hervorgehoben: Osaka, über Zuckerinversion; H irat a, Viskosität verdünnter alkoholischer Lösungen; $\mathrm{K}$ a ta y a ma, Anomalie der starken einwertigen Elektrolyte; Kaw a m u ra, Koagulation von Aluniniumhydroxyd durch Elektrolyte; Potdar, über die Verteilung von Silber zwischen Zink und Blei; Ikeda, Studien über die chemische Lösungstheorie I; von Yamamoto und Hirobe, phasentheoretische Stụdien über zwei binäre Systeme; O g a w a, vorläufige Mitteilung tiber ein neues Element (Nipponium) und ein zweites, dem Molybdän nahestehendes, sowie Andeutungen eines dritten. Die $\mathrm{I} 6$ in englischer und zwei in deutscher Sprache verfaßten Artikel geben deutlich das intensive Leben zu erkennen, welches in dem auch sonst so aktiven Volke der Japaner auf allen Gebieten der Chemie herrscht.

R. Abegg.

\title{
Eingegangene Bücher.
}

Le Bureau des Longitudes, Annuaire, pour l'an Igog. Verlag von Gauthier-Villars, Paris.

Friese, W., Die Asphalt- und Teerindustrie. Verlag von Dr. M. Jänecke, Hannover. 1908. Preis 5 Mk.

Kalender der Technischen Hochschulen. Verlag von J. A. Barth, Leipzig. 1908. Preis $2 \mathrm{Mk}$.

Stark, J., Jahrbuch der Radioaktivität und Elektronik. $\mathrm{V}, 3$. Verlag von $\mathbf{S}$. Hirzel, Leipzig. Igo8.

Breitfeld, Prof. Dr., Leitfaden für den Unterricht in der Naturlehre, Verlag von H. A, Ludwig Degner, Lelpzig. Preis I,50 Mk.
Abbilduugen zur Naturlehre. Verlag von H. A. Ludwig Degner, Leipzig. Igo8. Preis I Mk.

Scheel, K., Die Fortschritte der Physik im Jahre 1907, II. Verlag von Fr. Vieweg \& Sohn, Braunschweig.

Teichmann, Dr. H., Komprimierte und verflüssigte Gase. Verlag von Wilhelm Knapp, Halle a. S. Preis $6,80 \mathrm{Mk}$.

Vageler, Dr. P., Die mineralischen Nährstoffe der Pflanze. (Wissen und Können.) Verlag von J.A. Barth, Leipzig. Igo8. Preis 3 Mk. 